\title{
PHYSICOCHEMICAL AND ANTIMICROBIAL PROPERTIES OF COPAIBA OIL: IMPLICATIONS ON PRODUCT QUALITY CONTROL*
}

\author{
Renata G. Fonsecaํㅡㄹ Francisco M. Barros², Miriam A. Apel², Gilsane L. von Poser ${ }^{2}$, \\ João L. Andriolli ${ }^{1}$, Pedro C. Campos Filho ${ }^{3}$, Dhierlate F. Sousa ${ }^{3}$, Ivon P. Lobo ${ }^{4}$, \\ Aline O. Conceição ${ }^{1 凶}$
}

${ }^{1}$ Biological Science Department, State University of Santa Cruz - 16 km Jorge Amado Road, 45662-900, Ilheus, Bahia, Brazil

${ }^{2}$ Pharmaceutical Science Graduate Program, Federal University of Rio Grande do Sul -2752 Ipiranga Av., 90610-000, Porto Alegre, Rio Grande do Sul, Brazil

${ }^{3}$ Basic and Instrumental Studies Department, State University of Southeast Bahia BR Road 41503 Km, 45700-000, Itapetinga, Bahia, Brazil

${ }^{4}$ Exact and Technological Sciences Department, State University of Santa Cruz - $16 \mathrm{~km}$ Jorge Amado Road, 45662-900, Ilheus, Bahia, Brazil

\begin{abstract}
Background. The copaiba oil is a common natural product used in cosmetic industry and as a nutraceutical product. However, lack of quality control and scarce knowledge about its antimicrobial activity is a point of concern. The proposal of this study was to investigate the physicochemical properties and the antimicrobial activity of five commercial brands of copaiba oil.

Material and methods. Acidity and ester index, refractory index, solubility in alcohol, and thin layer chromatography were performed to verify the physicochemical properties of five commercial copaiba oils sold in local pharmacies. Ultra performance liquid chromatography coupled with diode-array detection and electrospray ionization quadrupole time-of-flight mass spectrometry (UPLC-DAD/ESI-Q-TOF-MS) was used to investigate diterpene acids while the volatile compounds were analysed by gas chromatography-mass spectrometry (GC-MS). Antibacterial and antifungal activities were also evaluated by agar diffusion technique; and minimal inhibitory concentration and maximal bactericidal concentration were defined for each sample and bacteria.

Results. The physical-chemical analysis revealed heterogeneity between all samples analysed. The A1 sample showed characteristics of copaiba oil and was mainly composed by hydrocarbon sesquiterpenes $(29.95 \%$ $\beta$-bisabolene, $25.65 \% Z$ - $\alpha$-bergamotene and 10.27\% $\beta$-cariophyllene). Among diterpene acids, the UPLCDAD/ESI-Q-TOF-MS data are compatible with presence of copalic and/or kolavenic acid (m/z 305 [M + $\mathrm{H}]^{+}$). Candida albicans was sensitive to almost all samples at high concentration and Saccaromyces. cerevisiae showed sensitivity to A1 sample at $100 \mathrm{mg} / \mathrm{mL}$. Although variable, all samples showed antibacterial activity. Significant activity was seen for A3 (19.0 \pm 0 and $15.6 \pm 0.5 \mathrm{~mm})$, A4 (16.6 \pm 0.5 and $15.6 \pm 0 \mathrm{~mm})$, and A5 (17.1 \pm 0 and $17.1 \pm 0 \mathrm{~mm}$ ) on Staphylococcus saprophyticus and $S$. aureus, respectively. All samples were active against Klebsiella pneumoniae showing $\geq 15 \mathrm{~mm}$ diameter halo inhibition; and only A2 was active against Eschirichia coli. Phytopatogens tested revealed resistance of Ralstonia solanacearum CGH12 to all samples and susceptibility of Xcv 112 strain of Xanthomonas campestris pv campestris to almost all samples. MIC and MMC showed bacteriostatic effect against clinical interest bacteria and bactericidal effect against phytopatogens.
\end{abstract}

\footnotetext{
*The authors are grateful to the Brazilian agencies CAPES and CNPq for financial support.

凶aoconceicao@uesc.br
} 


\begin{abstract}
Conclusion. The results from physicochemical analysis reinforce the fact that it is imperative to include simple conventional methods in the analysis of oil products. The analysis of copaiba oil gives safe products and purity which ensure products with quality. Also, since copaiba oil is an over-the-counter product the results indicate that pharmacosurveillance must be improved by the governmental regulation agency to avoid microorganism resistance selection and to achieve better international quality products.
\end{abstract}

Key words: nutraceutical product, microorganisms, antimicrobial action

\section{INTRODUCTION}

The copaiba oil is of great application in the industry to improve life time of food and it is a medicinal product widely used (Pieri et al., 2009; Sartoratto et al., 2004; Tempone et al., 2008; Veiga and Pinto, 2002). Several medicinal properties described for this oil have been scientifically proven, including antimicrobial, antiinflammatory, and tissue regeneration (Amaral et al., 2005; Correia et al., 2008; Estevão et al., 2009; Santos, 2008; Mendonça and Onofre, 2009; Vieira et al., 2008). However, its great usage, the standardization and quality control of copaiba oil are very difficult. Copaiba genus has very homogeneous external characteristics and the environment conditions can lead to different oil composition which contributes to oil variation (Barbosa et al., 2012; Biavatti et al., 2006). Besides, another problem in Brazilian oil production is the adulteration of products sold in the market (Barbosa et al., 2009; Veiga et al., 1997). Thus, although antimicrobial activity of oil has been described in the literature, the relation of this activity with physicochemical analysis of commercial copaiba oil has not been done. Thus, the objective of this study was to investigate the in vitro antimicrobial properties of commercial copaiba oils bought in local market associated to their physicochemical analysis.

\section{MATERIAL AND METHODS}

\section{Copaiba oil}

Five copaiba oils were purchased in local market from Ilheus and Itabuna, Bahia, in June 2013. Samples were within the validity period, labelled and tightly closed. Products were manipulated under aseptic conditions, weighted in $1.5 \mathrm{~mL}$ tubes, and dissolved in a dimethyl sulfoxide (DMSO)-sterile distilled water solution $(50: 50)$ to obtain biological test concentrations.
To perform physicochemical analysis, copaiba oil was used directly from the vessel. Samples were identified by letters and numbers: from A1 to A5.

\section{Physicochemical analysis}

The methods described in Vasconcelos and Goldinho (2002) were followed with some adaptation for all physicochemical analysis related to acidity index $(A I)$, ether index $(E I)$, and solubility in ethanol. Briefly, for $A I, 2 \mathrm{~g}$ of oil were weighted in $125 \mathrm{~mL}$ Becker and added of $25 \mathrm{~mL}$ an ethyl ether : ethyl alcohol (2:1) mixture. The $A I$ value was obtained by the equation $V \times N \times 56.1 / m$, where $V$ is $\mathrm{NaOH}$ volume in $\mathrm{mL}$ used for titration, $N$ is the normality of $\mathrm{NaOH}$ solution, and $m$ is the mass of each sample (g). For EI determination, the methodology described by Vasconcelos and Godinho (2002) with adaptations was also followed up. Briefly, $2 \mathrm{~g}$ of oil were weighed into a $125 \mathrm{~mL}$ flask where then $5 \mathrm{ml}$ of ethanol and 2 drops of phenolphthalein alcohol solution for performing titration with $0.1 \mathrm{~N} \mathrm{NaOH}$ were added. Then, to the titration of the resulting solution $20 \mathrm{~mL}$ of $4 \% \mathrm{KOH}$ were added under heating. After cooling, the solution received 2 more drops of alcoholic phenolphthalein solution. Then, new titration was carried out using $0.5 \mathrm{~N} \mathrm{HCl}$ solution. A titration without the oil sample was tested for comparison (blank). The EI values were obtained also using the equation $V \times N \times 56.1 / \mathrm{m}$, where $V$ is the difference between the volume of $\mathrm{HCl}$ solution spent in titration of blank and sample in milliliters, $N$ is the normality of the $\mathrm{HCl}$ solution and $m$ is weight of each sample $(\mathrm{g})$. The solubility in ethanol was performed using $1 \mathrm{~mL}$ of each oil sample to which were added $2 \mathrm{~mL}$ of ethanol analytical grade under agitation for 5 seconds. Samples were classified as soluble, insoluble, and partially soluble. The refractive index was 
performed for each sample using Abbé (Optronics Inc., Precision Instruments, Germany).

Thin layer chromatography was performed with $20 \times 20 \mathrm{~cm}$ aluminum plates covered with silica gel 60 containing fluorescent indicator Alugram Sil G/UV 254 (Macherey-Nagel, Germany). Elution was done with $n$-hexane:ethyl acetate $(9: 1)$ and visualization was done by spraying with $20 \%$ sulfuric acid solution and heating. Color aspect of spots and retention factor $\left(R_{f}\right)$ was measured to compare with soybean oil, a common oil found in adulteration of copaiba oil (Barbosa et al., 2009; Vasconcelos and Goldinho, 2002).

The presence of diterpene acids was investigated by ultra performance liquid chromatography coupled with diode-array detection (UPLC-DAD) and electrospray ionization quadrupole time-of-flight mass spectrometry (ESI-Q-TOF-MS) using Waters Acquity UPLC system connected to Waters Q-TOF Premier mass spectrometer (Waters, Milford, CA, USA). A slightly modified method of high performance liquid chromatography (HPLC) (Souza et al., 2013) was successfully transferred to UPLC with aid of ACQUITY UPLC ${ }^{\mathbb{R}}$ Columns calculator (Waters, Milford, CA, USA). Separations were carried out on a Hypersyl Gold C18 column $(1.9 \mu \mathrm{m}, 2.1 \mathrm{~mm} \times 100 \mathrm{~mm}$; Themo Scientific, USA). The mobile phase consisted of $90 \%$ acetonitrile (Merck, Germany), 10\% water (Milli-Q) and 1\% (v/v) formic acid ( $96 \%$, Tedia, USA). The analyses were performed at $25^{\circ} \mathrm{C}$ and at a flow rate of $0.47 \mathrm{~mL} / \mathrm{min}$ over $5 \mathrm{~min}$. The oils were dissolved in methanol $(500 \mu \mathrm{g} / \mathrm{mL})$ and an aliquot of $8 \mu \mathrm{L}$ of each sample was injected. Mass detection $(\mathrm{m} / \mathrm{z}=50$ to 1000$)$ was carried out in positive mode and data were processed with MassLynx V4.1 software. Nitrogen and Argon were used as the nebulizer and collision gases, respectively. Other conditions: DAD, 200 to $400 \mathrm{~nm}$; ESI capillary voltage, $+3000 \mathrm{~V}$; source and desolvation temperatures, 100 and $250^{\circ} \mathrm{C}$, respectively; desolvation and cone gas flow, 380 and $50 \mathrm{~L} / \mathrm{h}$, respectively; sample cone voltage and collision energy, $30 \mathrm{~V}$ and $4 \mathrm{eV}$, respectively.

Additionally, the A1 sample was diluted in ethyl ether $(2: 100 \mathrm{v} / \mathrm{v})$ and submitted to analysis by gas chromatography-mass spectrometry (GC-MS; Shimadzu QP5000), using a capillary column of fused silica Durabond-DB5 $(30 \mathrm{~m} \times 0.25 \mathrm{~mm} \times 0.25 \mu \mathrm{m})$. Temperature program was 60 to $300^{\circ} \mathrm{C}$ at $3^{\circ} \mathrm{C} / \mathrm{min}$. The injector and detector temperature was set at 220 and $250^{\circ} \mathrm{C}$, respectively. Carrier gas was helium at a flow rate of $1 \mathrm{~mL} / \mathrm{min}$. The compounds were identified by comparison of retention index, determined by linear interpolation relative to retention times of a homologous series of $n$-alkanes, and mass spectra (electronic impact, $70 \mathrm{eV}$ ) with the literature data (Adams, 2009) or National Institute of Technology and Standards database (NIST 62 and NIST 12). The relative percentage of each component was calculated from GC peak areas.

\section{Microorganisms}

For this study, clinical interest bacteria and yeast and phytopathogens were used. Bacteria were kindly provided by Pedro Costa Campos from the Biological Sciences Department from Bahia State University and yeasts were kindly provided by Dr. Sydney Hartz Alves from Mycological Research Laboratory (LAPEME) from Federal University of Santa Maria. The microorganisms used were of clinical interest (Enterococcus faecalis, ATCC31299; Escherichia coli, ATCC35218; Klebsiella pneumonia, ATCC700603; Pseudomonas aeruginosa, ATCC27853; Staphylococcus aureus, ATCC25921; Staphylococcus saprophyticus, ATCC35552; Candida albicans, ATCC 14057; C. glabrata, ATCC 2301; C. parapsilosis, ATCC 22018; C. krusei, ATCC 6258; Saccaromyces cerevisiae, ATCC 2601) and phytopathogens (Acidovorax citrulli, Aac. 1.12; Pectobacterium caratovorum subsp. caratovorum, Pcc23; Ralstonia solanacearum, CGH12; Ralstonia solanacearum, B19; Xanthomonas campestris pv campestres, Xcv112; Xanthomonas campestris pv campestris, Xcc56).

\section{Agar gel diffusion technique}

For qualitative analysis, the well agar gel diffusion technique was used. For that, bacterial and yeast inoculums were prepared at an equivalent of 0.5 of McFarland scale (approx. cell density of $1.5 \cdot 10^{8} \mathrm{CFU} / \mathrm{mL}$ ) and spread with sterile swab all over the Petri dishes containing nutrient or Saboureaud dextrose agar. The oils were prepared immediately before the test at 50,100 , and $1,000 \mathrm{mg} / \mathrm{mL}$. After bacterial and yeast culture in Petri dishes, holes with $6 \mathrm{~mm}$ diameter were done in the agar where $50 \mu \mathrm{L}$ of samples and controls were applied. Controls consisted of chloramphenicol $(30 \mu \mathrm{g} / \mathrm{mL})$, ketoconazole $(50 \mu \mathrm{g} / \mathrm{mL})$, chlorexidine $(1 \%)$, sterile distilled water and DMSO (50:50). The inverted plates were incubated overnight at $37^{\circ} \mathrm{C}$ in a humidified 
chamber to avoid evaporation of the medium. All tests were done in triplicate and repeated at least twice.

To analyse the antibacterial activity, inhibition halo $\geq 14 \mathrm{~mm}$ at $\leq 100 \mathrm{mg} / \mathrm{mL}$ were used as criteria to consider a sample as antibacterial. Following criteria established by Pedroso et al. (2014), the inhibition halo of $\geq 10 \mathrm{~mm}$ was used as the criterion to consider a sample as antifungal.

\section{Determination of the Minimum Inhibitory Concentration (MIC) and Minimum Microbicidal Concentration (MMC)}

The MIC and MMC were performed only for bacterial samples. The determination of MIC was done using a micro-dilution assay. Briefly, after filtration in $0.22 \mu \mathrm{m}$ pore membrane, oils from 3.9 to $500 \mathrm{mg} / \mathrm{mL}$ were prepared in Müeller-Hinton broth and $90 \mu \mathrm{L}$ of samples were distributed in plate wells. Additionally, $10 \mu \mathrm{L}$ of bacterial suspension standardized at 0.5 of McFarland scale, was added to each well except in broth sterility controls. Controls consisted of chloramphenicol $(30 \mu \mathrm{g} / \mathrm{mL})$ and DMSO (50:50). After $24 \mathrm{~h}$ culture at $37^{\circ} \mathrm{C}, 30 \mu \mathrm{L}$ of Rezasurin (Sigma-Aldrich, Brazil) were applied given $0.01 \%$ final concentration. Pink colour wells were considered positive and purple colour wells were considered negative. The MMC were then performed by taking $5 \mu \mathrm{L}$ of CIM positive samples and inoculating Petri dishes containing agar Müller-Hinton. The plates were incubated at $37^{\circ} \mathrm{C}$ in humidified chamber. After $24 \mathrm{~h}$, bacterial colonies were counted. The bacterial growth at lowest concentration was considered the MMC of the oil.

\section{RESULTS}

\section{Physicochemical analyses}

The physicochemical analysis revealed heterogeneity between all samples analysed. The A1 showed in natura copaiba oil characteristics and A5 showed strongly characteristics of soybean oil adulteration (Table 1) confirmed by the presence of an orange spot at $R_{f} 4.2$, equal to soybean oil (Fig. 1).

According to UPLC-DAD/ESI-Q-TOF-MS all samples presented one peak at $1.74 \pm 0.01 \mathrm{~min}$ (Fig. 2) consistent with m/z $305[\mathrm{M}+\mathrm{H}]^{+}$(Fig. 3) of copalic acid and/or kolavenic acid (Messiano et al., 2008; Marchesini et al., 2009). The A1 sample was selected to GC-MS analysis and presented $\beta$-bisabolene (29.95\%), $Z$ - $\alpha$ -bergamotene (25.65\%) and $\beta$-cariophyllene (10.27\%) as major compound (Table 2).

\section{Antimicrobial activity tests}

The antifungal activity revealed that however at high concentration $(1,000 \mathrm{mg} / \mathrm{mL})$, C. albicans was sensitive to almost all samples with exception of sample A1. It is worth to note that the best response was for S. cerevisiae which showed sensitivity to A4 sample at $100 \mathrm{mg} / \mathrm{mL}$ (Table 3).

Table 1. Physicochemical analysis of commercial samples (A1 to A5) of copaiba oil and soybean oil

\begin{tabular}{lcccl}
\hline Oil sample & AI & $\begin{array}{c}\text { EI } \\
\mathrm{mg} \mathrm{KOH} / \mathrm{g}\end{array}$ & RI & \multicolumn{1}{c}{ SE } \\
\hline A1 & 80.04 & 23 & 1.5020 & soluble \\
A2 & 42.82 & 20 & 1.5034 & partially soluble \\
A3 & 24.90 & 16 & 1.4986 & partially soluble \\
A4 & 38.93 & 41 & 1.4920 & partially soluble \\
A5 & 10.39 & 105 & 1.4851 & insoluble \\
Soybean oil & - & 181 & 1.4747 & insoluble \\
\hline
\end{tabular}

$\mathrm{AI}$ - acidity index. EI - ether index. RI - refractive index. SE - solubility in ethanol. Standard values for in natura copaiba oil: AI - $80 \mathrm{mg} \mathrm{KOH} / \mathrm{g}$, EI - $23 \mathrm{mg} \mathrm{KOH} / \mathrm{g}$, RI 1.50284 to 1.50786 , $\mathrm{SE}$ - soluble. 
Fonseca, R. G., Barros, F. M., Apel, M. A., von Poser, G. L., Andriolli, J. L., Filho, P. C. C., Sousa, D. F., Lobo, I. P., Conceição, A. O. (2015). Physicochemical and antimicrobial properties of copaiba oil: implications on product quality control. Acta Sci. Pol. Technol. Aliment., 14(3), 215-225. DOI: 10.17306/J.AFS.2015.3.23

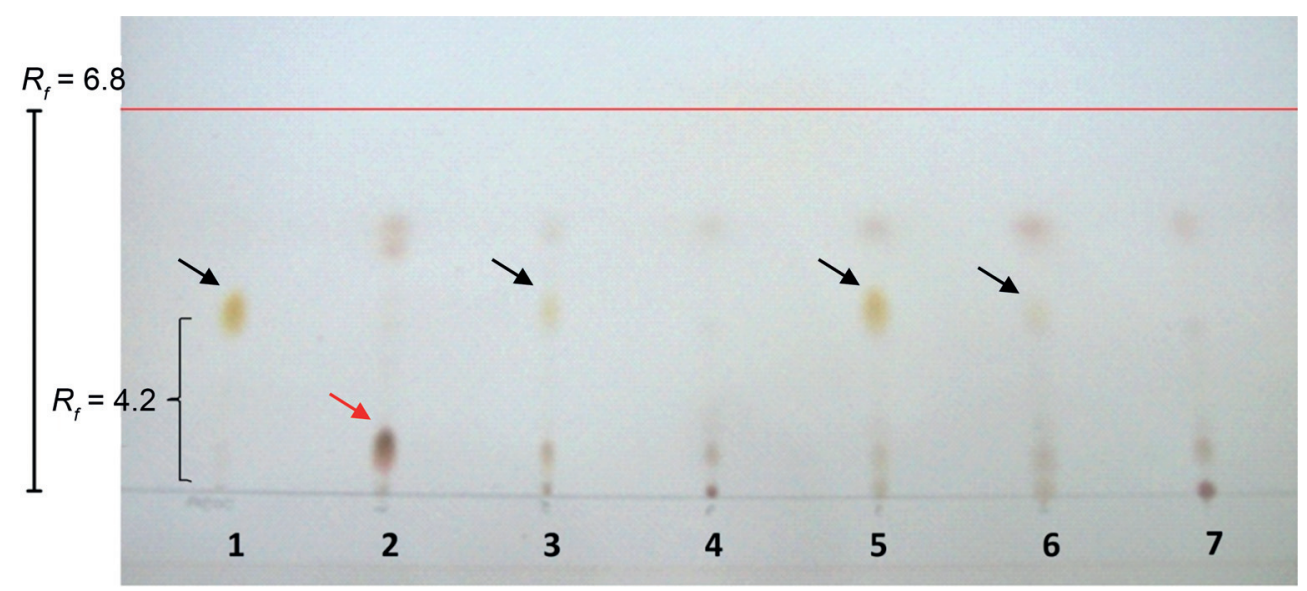

Fig. 1. Thin layer chromatography profile of oil-resin commercial samples and soybean oil: 1 soybean oil, 2 - A1, 3 - Af, 4-A2, 5-A5, 6-A3, 7 - A4. Red arrow - characteristic of copaiba oil-resin spot; black arrow - characteristic of copaiba oil-resin spot, red line - upper limit of mobile phase, $R_{f}$ - retention factor

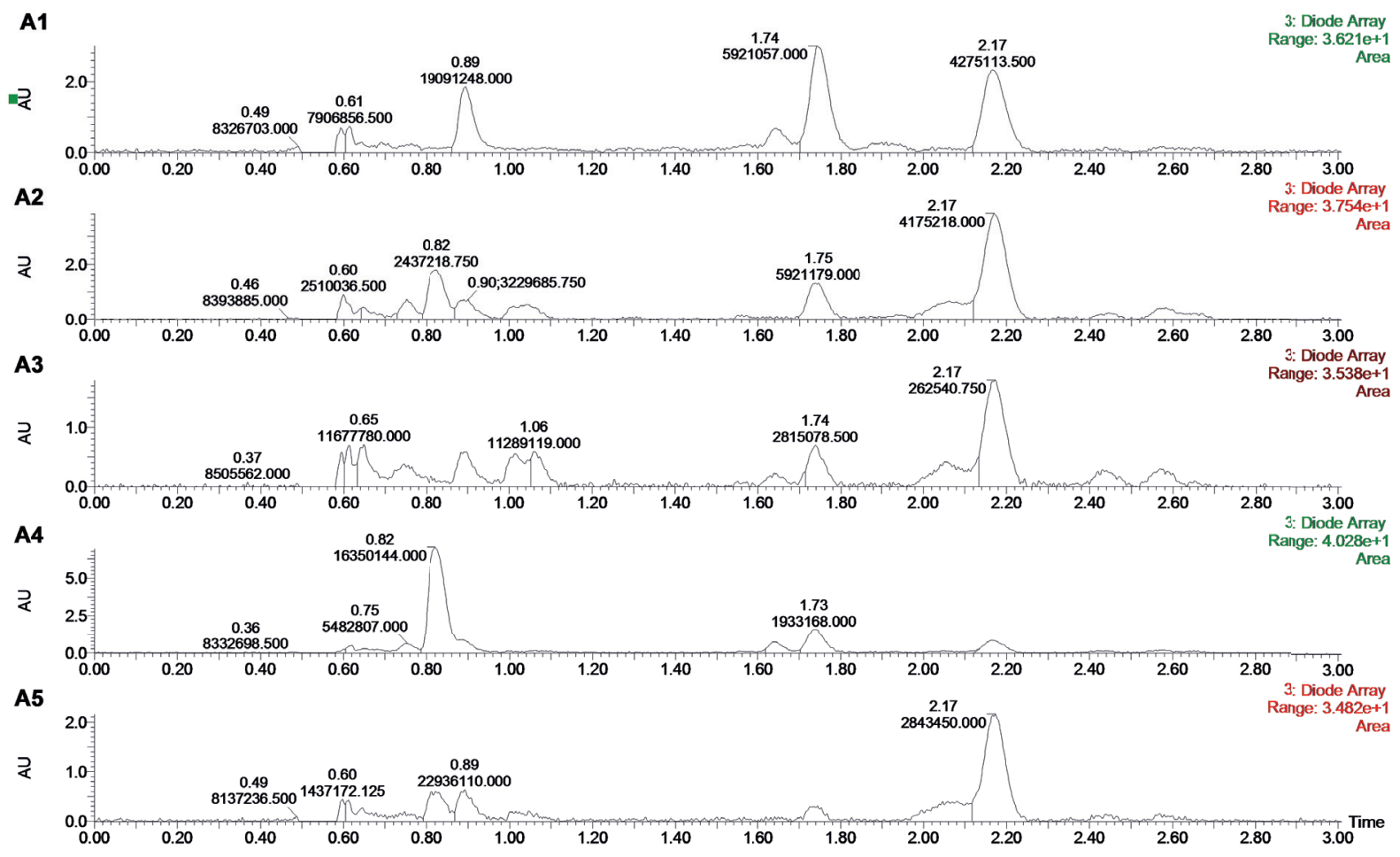

Fig. 2. UPLC-UV (220 nm) profile of copaiba oil commercial samples 


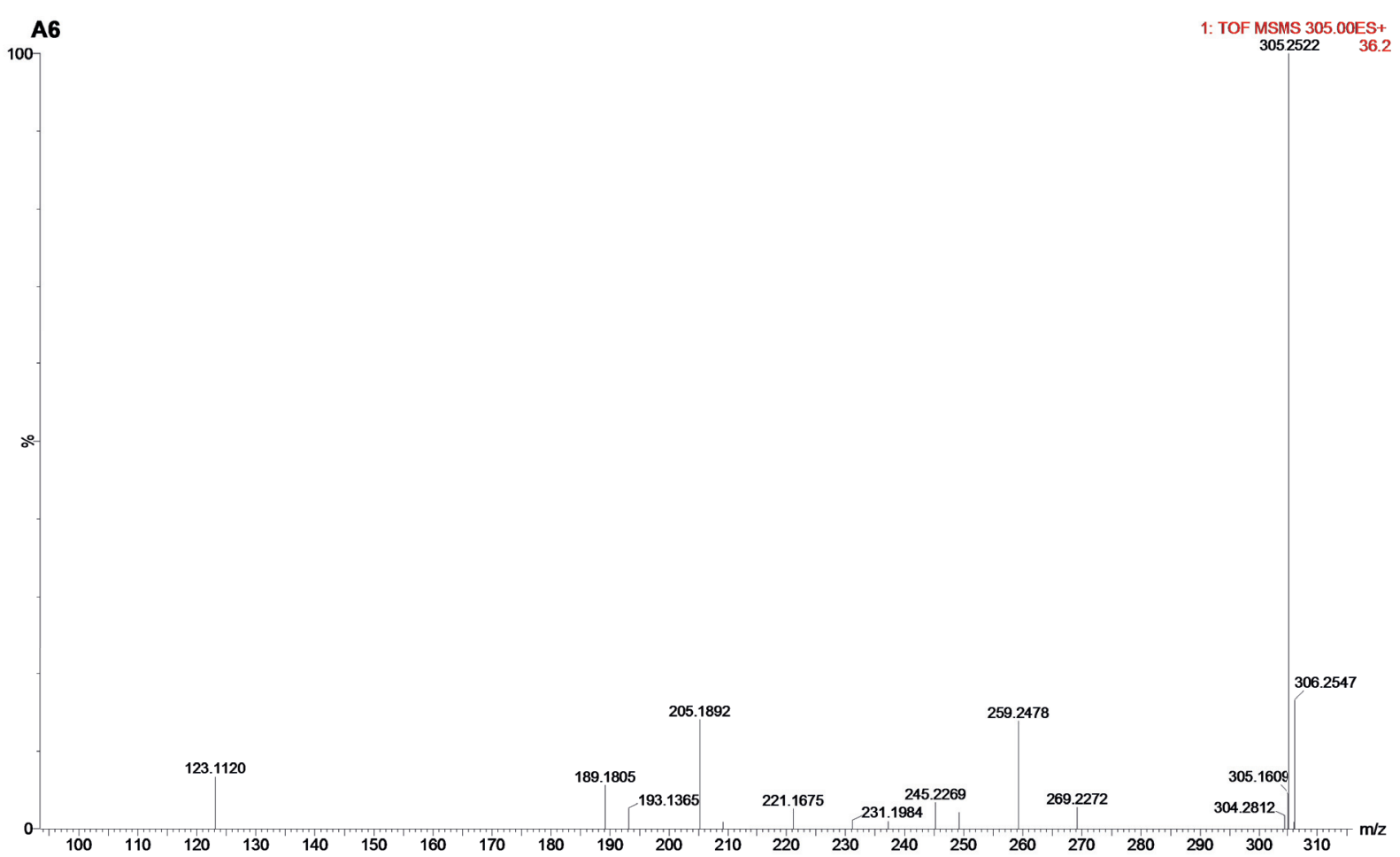

Fig. 3. UPLC-ESI-Q-TOF-MS of A1 sample. MS/MS (ESI: $15 \mathrm{eV}$ ) of peak with $m / z 305[\mathrm{M}+\mathrm{H}]^{+}$

Table 2. Antifungal activity of five commercial copaiba oil (A1 to A5) by agar diffusion technique - well variable

\begin{tabular}{|c|c|c|c|c|c|c|c|c|c|c|c|c|c|c|c|}
\hline \multirow[t]{2}{*}{$\begin{array}{c}\text { Copaiba oil } \\
\mathrm{mg} / \mathrm{mL}\end{array}$} & \multicolumn{3}{|c|}{$\begin{array}{l}\text { Candida albicans } \\
\text { ATCC } 14057\end{array}$} & \multicolumn{3}{|c|}{$\begin{array}{l}\text { C. glabrata } \\
\text { ATCC } 2301\end{array}$} & \multicolumn{3}{|c|}{$\begin{array}{c}\text { C. krusei } \\
\text { ATCC } 6258\end{array}$} & \multicolumn{3}{|c|}{$\begin{array}{c}\text { C. parapsilosis } \\
\text { ATCC } 22018\end{array}$} & \multicolumn{3}{|c|}{$\begin{array}{c}\text { Sacharomyces } \\
\text { cerevisiae } \\
\text { ATCC } 2601\end{array}$} \\
\hline & 50 & 100 & 1000 & 50 & 100 & 1000 & 50 & 100 & 1000 & 50 & 100 & 1000 & 50 & 100 & 1000 \\
\hline A1 & $\mathrm{R}$ & $\mathrm{R}$ & $\mathrm{R}$ & $\mathrm{R}$ & $9.0 \pm 0$ & $\mathrm{R}$ & $\mathrm{R}$ & $\mathrm{R}$ & $\mathrm{R}$ & $\mathrm{R}$ & $\mathrm{R}$ & $\mathrm{R}$ & $\mathrm{R}$ & $9.0 \pm 0$ & $\mathrm{R}$ \\
\hline $\mathrm{A} 2$ & $\mathrm{R}$ & $\mathrm{R}$ & $10.0 \pm 0$ & $\mathrm{R}$ & $\mathrm{R}$ & $\mathrm{R}$ & $\mathrm{R}$ & $\mathrm{R}$ & $\mathrm{R}$ & $\mathrm{R}$ & $\mathrm{R}$ & $\mathrm{R}$ & $\mathrm{R}$ & $\mathrm{R}$ & $\mathrm{R}$ \\
\hline A3 & $\mathrm{R}$ & $\mathrm{R}$ & $10.0 \pm 0$ & $\mathrm{R}$ & $\mathrm{R}$ & $\mathrm{R}$ & $\mathrm{R}$ & $\mathrm{R}$ & $\mathrm{R}$ & $\mathrm{R}$ & $\mathrm{R}$ & $\mathrm{R}$ & $\mathrm{R}$ & $\mathrm{R}$ & $\mathrm{R}$ \\
\hline A4 & $\mathrm{R}$ & $\mathrm{R}$ & $10.0 \pm 0$ & $\mathrm{R}$ & $\mathrm{R}$ & $\mathrm{R}$ & $\mathrm{R}$ & $\mathrm{R}$ & $\mathrm{R}$ & $\mathrm{R}$ & $\mathrm{R}$ & $\mathrm{R}$ & $\mathrm{R}$ & $13.0 \pm 0$ & $\mathrm{R}$ \\
\hline A5 & $\mathrm{R}$ & $\mathrm{R}$ & $10.0 \pm 0$ & $\mathrm{R}$ & $\mathrm{R}$ & $\mathrm{R}$ & $\mathrm{R}$ & $\mathrm{R}$ & $\mathrm{R}$ & $\mathrm{R}$ & $\mathrm{R}$ & $\mathrm{R}$ & $\mathrm{R}$ & $\mathrm{R}$ & $\mathrm{R}$ \\
\hline CHL 1\% & & & & & & & & & & & & & & 21 & \\
\hline $\begin{array}{l}\mathrm{KTZ} \\
50 \mu \mathrm{g} / \mathrm{mL}\end{array}$ & & 35 & & & 30 & & & 30 & & & 35 & & & & \\
\hline $\begin{array}{l}\text { DMSO } \\
(50: 50)\end{array}$ & & $\mathrm{R}$ & & & $\mathrm{R}$ & & & $\mathrm{R}$ & & & $\mathrm{R}$ & & & $\mathrm{R}$ & \\
\hline
\end{tabular}

$\mathrm{R}$ - Resistant. Agar holes measured $6 \mathrm{~mm}$. The diameter of the inhibition halo is expressed in millimetre. Values represent means and standard deviation of three independent experiments. $\mathrm{CHL}$ - chlorhexidine, $\mathrm{KTZ}$ - ketoconazole, DMSO - dimethyl sulfoxide. 
Fonseca, R. G., Barros, F. M., Apel, M. A., von Poser, G. L., Andriolli, J. L., Filho, P. C. C., Sousa, D. F., Lobo, I. P., Conceição, A. O. (2015). Physicochemical and antimicrobial properties of copaiba oil: implications on product quality control. Acta Sci. Pol. Technol. Aliment., 14(3), 215-225. DOI: 10.17306/J.AFS.2015.3.23

Table 3. Antibacterial activity of five commercial copaiba oils (A1 to A5) by agar diffusion technique - well variable against clinical importance bacteria

\begin{tabular}{|c|c|c|c|c|c|c|c|c|c|c|c|c|c|c|c|}
\hline $\begin{array}{c}\text { Sample/ } \\
\text { Microorganism }\end{array}$ & & A1 & & & $\mathrm{A} 2$ & & & A3 & & & A4 & & & A5 & \\
\hline $\mathrm{mg} / \mathrm{mL}$ & 50 & 100 & 1000 & 50 & 100 & 1000 & 50 & 100 & 1000 & 50 & 100 & 1000 & 50 & 100 & 1000 \\
\hline $\begin{array}{l}\text { E. coli } \\
\text { (ATCC 35218) }\end{array}$ & $\begin{array}{l}9.3 \\
\pm 0\end{array}$ & $\begin{array}{l}11.0 \\
\pm 0\end{array}$ & $\begin{array}{l}11.6 \\
\pm 0.5\end{array}$ & $\begin{array}{l}12.0 \\
\pm 0\end{array}$ & $\begin{array}{c}14.0 \\
\pm 0\end{array}$ & $\begin{array}{l}16.6 \\
\pm 0.5\end{array}$ & $\mathrm{R}$ & $\mathrm{R}$ & $\mathrm{R}$ & $\mathrm{R}$ & $\mathrm{R}$ & $\mathrm{R}$ & $\mathrm{R}$ & $\mathrm{R}$ & $\mathrm{R}$ \\
\hline $\begin{array}{l}\text { E. faecalis } \\
\text { (ATCC31299) }\end{array}$ & $\begin{array}{l}11.0 \\
\pm 0\end{array}$ & $\begin{array}{l}10.0 \\
\pm 0\end{array}$ & $\begin{array}{l}19.0 \\
\pm 0\end{array}$ & $\begin{array}{l}10.0 \\
\pm 0\end{array}$ & $\begin{array}{l}12.0 \\
\pm 0\end{array}$ & $\begin{array}{l}13.3 \\
\pm 0.5\end{array}$ & $\begin{array}{l}12.0 \\
\pm 0\end{array}$ & $\begin{array}{l}13.6 \\
\pm 0.5\end{array}$ & $\begin{array}{c}13.3 \\
\pm 0\end{array}$ & $\begin{array}{l}10.0 \\
\pm 0\end{array}$ & $\begin{array}{l}10.0 \\
\pm 0\end{array}$ & $\begin{array}{c}10 \\
\pm 0.5\end{array}$ & $\mathrm{R}$ & $\mathrm{R}$ & $\begin{array}{c}9.6 \\
\pm 0.5\end{array}$ \\
\hline $\begin{array}{l}\text { K. pneumoniae } \\
\text { (ATCC700603) }\end{array}$ & $\begin{array}{c}16.0 \\
\pm 0\end{array}$ & $\begin{array}{c}16.0 \\
\pm 0\end{array}$ & $\begin{array}{l}19.0 \\
\pm 0\end{array}$ & $\begin{array}{c}16.0 \\
\pm 0\end{array}$ & $16 \pm 0$ & $\begin{array}{c}15.0 \\
\pm 0\end{array}$ & $\begin{array}{c}9.6 \\
\pm 0.5\end{array}$ & $\begin{array}{l}15.9 \\
\pm 0.5\end{array}$ & $\begin{array}{l}13.6 \\
\pm 0.5\end{array}$ & $\begin{array}{l}13.6 \\
\pm 0.5\end{array}$ & $\begin{array}{c}15.0 \\
\pm 0\end{array}$ & $15 \pm 0$ & $\begin{array}{l}15.0 \\
\pm 0.5\end{array}$ & $\begin{array}{l}15.5 \\
\pm 0.5\end{array}$ & $\begin{array}{c}9.6 \\
\pm 0.5\end{array}$ \\
\hline $\begin{array}{l}\text { P. aeruginosa } \\
\text { (ATCC } 27853 \text { ) }\end{array}$ & $\begin{array}{c}10.0 \\
\pm 0\end{array}$ & $\begin{array}{l}11.0 \\
\pm 1.1\end{array}$ & $\begin{array}{c}19.0 \\
\pm 0\end{array}$ & $\mathrm{R}$ & $\begin{array}{c}12 \\
\pm 0.5\end{array}$ & $\begin{array}{c}13.0 \\
\pm 0\end{array}$ & $\begin{array}{l}12.0 \\
\pm 0\end{array}$ & $\begin{array}{l}13.6 \\
\pm 0.5\end{array}$ & $\begin{array}{c}12.0 \\
\pm 0\end{array}$ & $\begin{array}{c}13.0 \\
\pm 0\end{array}$ & $\begin{array}{l}10.0 \\
\pm 0\end{array}$ & $\begin{array}{c}13.0 \\
\pm 0\end{array}$ & $\begin{array}{l}11.66 \\
\pm 0.5\end{array}$ & $\begin{array}{c}13.0 \\
\pm 0\end{array}$ & $\begin{array}{c}9.6 \\
\pm 0.5\end{array}$ \\
\hline $\begin{array}{l}\text { S. aureus } \\
\text { (ATCC 25921) }\end{array}$ & $\mathrm{R}$ & $\begin{array}{c}10.0 \\
\pm 0\end{array}$ & $\begin{array}{c}15.0 \\
\pm 0\end{array}$ & $\begin{array}{c}12.0 \\
\pm 0\end{array}$ & $\begin{array}{c}14.0 \\
\pm 0\end{array}$ & $\begin{array}{l}19.0 \\
\pm 0\end{array}$ & $\begin{array}{l}12.6 \\
\pm 0.5\end{array}$ & $\begin{array}{l}15.6 \\
\pm 0.5\end{array}$ & $\begin{array}{c}16.0 \\
\pm 0\end{array}$ & $\begin{array}{l}16.0 \\
\pm 0\end{array}$ & $\begin{array}{c}15.6 \\
\pm 0\end{array}$ & $\begin{array}{c}15 \\
\pm 0.5\end{array}$ & $\begin{array}{l}13.6 \\
\pm 0.5\end{array}$ & $\begin{array}{c}17.0 \\
\pm 0\end{array}$ & $\begin{array}{c}20.1 \\
\pm 0\end{array}$ \\
\hline $\begin{array}{l}\text { S. saprophyticus } \\
\text { (ATCC 35552) }\end{array}$ & $\begin{array}{c}13.0 \\
\pm 0\end{array}$ & $\begin{array}{l}19.3 \\
\pm 1.1\end{array}$ & $\begin{array}{l}16.6 \\
\pm 0.5\end{array}$ & $\begin{array}{c}9.6 \\
\pm 0.5\end{array}$ & $\begin{array}{c}9.6 \\
\pm 0.5\end{array}$ & $\begin{array}{c}9.6 \\
\pm 0.5\end{array}$ & $\begin{array}{l}16.6 \\
\pm 0.5\end{array}$ & $\begin{array}{l}19.0 \\
\pm 0\end{array}$ & $\begin{array}{l}19.0 \\
\pm 0\end{array}$ & $\begin{array}{l}15.6 \\
\pm 0.5\end{array}$ & $\begin{array}{l}16.6 \\
\pm 0.5\end{array}$ & $\begin{array}{l}19.6 \\
\pm 0.5\end{array}$ & $\begin{array}{l}16.6 \\
\pm 0.5\end{array}$ & $\begin{array}{c}17.1 \\
\pm 0\end{array}$ & $\begin{array}{c}17.0 \\
\pm 0\end{array}$ \\
\hline
\end{tabular}

$\mathrm{R}$ - Resistant. No halo formation.

Although variable, all samples showed antibacterial activity taking into consideration the criteria established for this study. Through agar diffusion technique, significant activity was seen for A3 $(19.0 \pm 0$ and $15.6 \pm 0.5 \mathrm{~mm}), \mathrm{A} 4(16.6 \pm 0.5$ and $15.6 \pm 0 \mathrm{~mm})$, and A5 (17.1 \pm 0 and $17.0 \pm 0 \mathrm{~mm})$ on S. saprophyticus and $S$. aureus, respectively. All samples were active against $K$. pneumoniae showing $\geq 15 \mathrm{~mm}$ diameter halo inhibition; and in accordance with the criteria used; only A2 was active against $E$. coli (Table 4).

In relation to phytopatogens, $R$. solanacearum CGH12 was resistant to all samples. Conversely, Xcv112 strain of $X$. campestris pv campestris was sensible to almost all samples ( $\geq 15.0 \pm 1.0 \mathrm{~mm}$; Table 4$)$.

Minimum Inhibitory Concentration (MIC) and MMC showed bacteriostatic effect against clinical interest bacteria and bacteriostatic and bactericidal effect against phytopatogens (Table 5).

\section{DISCUSSION}

The importance of copaiba oil for folk medicine and its standardization and quality control difficulties has been a point of interested. In this work the complemented data concerning copaiba oil confirmed the chemical differences between samples sold in the market and their influence on the antimicrobial property of the products in vitro. All samples tested in this work confirmed the antimicrobial potential of copaiba oil. The antifungal activity results showed weak action on Candida yeast as shown by Deus et al. (2011) being considered a good antifungal product only for $S$. cerevisiae. In relation to antibacterial activity, the results were a promise regarding therapeutic aspects since the oil proved to inhibit bacterial samples at lower concentrations with attention to the action against $S$. saprophyticus (ATTC35552) by diffusion agar technique. Other reports indicated the in natura copaiba oil extract of identified species such as $C$. reticulata (Oliveira et al., 2006; Santos et al., 2008), C. multijuga Hayne (Deus et al., 2011; Pieri et al., 2012) antimicrobial potential against Gram positive bacteria, including action against Methicillin-resistant Staphylococcus aureus (MRSA). However, these results indicate antimicrobial action, this copaiba oil effect seemed to be Gram positive restrain spectrum, since no action against Gram negative bacteria was reported. Conversely, our results reveal that commercialized oil can also act against Gram negative bacteria. Besides the antimicrobial action, this study revealed physicochemical differences between 
Fonseca, R. G., Barros, F. M., Apel, M. A., von Poser, G. L., Andriolli, J. L., Filho, P. C. C., Sousa, D. F., Lobo, I. P., Conceição, A. O. (2015). Physicochemical and antimicrobial properties of copaiba oil: implications on product quality control. Acta Sci. Pol. Technol. Aliment., 14(3), 215-225. DOI: 10.17306/J.AFS.2015.3.23

Table 4. Antibacterial activity of five commercial copaiba oils (A1 to A5) by agar diffusion technique - well variable against phytopathogens

\begin{tabular}{|c|c|c|c|c|c|c|c|c|c|c|c|c|c|c|c|}
\hline $\begin{array}{c}\text { Sample/ } \\
\text { Microorganism }\end{array}$ & & A1 & & & A2 & & & A3 & & & A4 & & & A5 & \\
\hline $\mathrm{mg} / \mathrm{mL}$ & 50 & 100 & 1000 & 50 & 100 & 1000 & 50 & 100 & 1000 & 50 & 100 & 1000 & 50 & 100 & 1000 \\
\hline $\begin{array}{l}\text { A. citrulli } \\
\text { (Aac. } 1.12)\end{array}$ & $\mathrm{R}$ & $\mathrm{R}$ & $\begin{array}{l}10.6 \\
\pm 0.5\end{array}$ & $\begin{array}{r}9.6 \\
\pm 0.5\end{array}$ & $\begin{array}{l}11.6 \\
\pm 0.5\end{array}$ & $\begin{array}{l}16.6 \\
\pm 1.5\end{array}$ & $\begin{array}{r}9.6 \\
\pm 0.5\end{array}$ & $\begin{array}{l}11.6 \\
\pm 0.5\end{array}$ & $\begin{array}{l}16.6 \\
\pm 1.5\end{array}$ & $\begin{array}{l}10.6 \\
\pm 0.5\end{array}$ & $\begin{array}{l}13.6 \\
\pm 0.5\end{array}$ & $\begin{array}{l}18.6 \\
\pm 0.5\end{array}$ & $\mathrm{R}$ & $\mathrm{R}$ & $\begin{array}{l}12.0 \\
\pm 1.0\end{array}$ \\
\hline $\begin{array}{l}\text { P. caratovorum } \\
\text { subsp. carato- } \\
\text { vorum }(\operatorname{Pcc} 23)\end{array}$ & $\begin{array}{l}10.0 \\
\pm 1.0\end{array}$ & $\begin{array}{l}10.6 \\
\pm 0.5\end{array}$ & $\begin{array}{l}13.0 \\
\pm 1.0\end{array}$ & $\begin{array}{l}12.6 \\
\pm 0.5\end{array}$ & $\begin{array}{l}11.6 \\
\pm 0.5\end{array}$ & $\begin{array}{l}15.6 \\
\pm 0.5\end{array}$ & $\begin{array}{l}10.3 \\
\pm 1.1\end{array}$ & $\begin{array}{l}10.0 \\
\pm 1.0\end{array}$ & $\begin{array}{l}13.6 \\
\pm 0.5\end{array}$ & $\begin{array}{l}10.6 \\
\pm 0.5\end{array}$ & $\begin{array}{l}14.6 \\
\pm 0.5\end{array}$ & $\begin{array}{l}14.6 \\
\pm 0.5\end{array}$ & $\begin{array}{r}9.6 \\
\pm 0.5\end{array}$ & $\begin{array}{l}14.0 \\
\pm 1.0\end{array}$ & $\begin{array}{l}16.6 \\
\pm 0.5\end{array}$ \\
\hline $\begin{array}{l}\text { R. solanace- } \\
\text { arum (B19) }\end{array}$ & $\begin{array}{l}12.0 \\
\pm 1.0\end{array}$ & $\begin{array}{l}14.0 \\
\pm 1.0\end{array}$ & $\begin{array}{l}15.0 \\
\pm 1.0\end{array}$ & $\begin{array}{r}9.6 \\
\pm 0.5\end{array}$ & $\begin{array}{r}9.6 \\
\pm 0.5\end{array}$ & $\begin{array}{r}9.6 \\
\pm 0.5\end{array}$ & $\begin{array}{l}14.3 \\
\pm 1.0\end{array}$ & $\begin{array}{l}14.0 \\
\pm 1.0\end{array}$ & $\begin{array}{l}13.6 \\
\pm 1.0\end{array}$ & $\begin{array}{l}13.6 \\
\pm 0.5\end{array}$ & $\begin{array}{l}20.6 \\
\pm 0.5\end{array}$ & $\begin{array}{l}25.6 \\
\pm 0.5\end{array}$ & $\begin{array}{l}13.0 \\
\pm 1.0\end{array}$ & $\begin{array}{l}18.0 \\
\pm 1.0\end{array}$ & $\begin{array}{l}18.6 \\
\pm 0.5\end{array}$ \\
\hline $\begin{array}{l}\text { R. solanace- } \\
\text { arum }(\mathrm{CGH} 12)\end{array}$ & $\mathrm{R}$ & $\mathrm{R}$ & $\mathrm{R}$ & $\mathrm{R}$ & $\mathrm{R}$ & $\mathrm{R}$ & $\mathrm{R}$ & $\mathrm{R}$ & $\mathrm{R}$ & $\mathrm{R}$ & $\mathrm{R}$ & $\mathrm{R}$ & $\mathrm{R}$ & $\mathrm{R}$ & $\mathrm{R}$ \\
\hline $\begin{array}{l}\text { X. campestris } \\
\text { PV campestris } \\
(\mathrm{Xcc} 56)\end{array}$ & $\begin{array}{l}12.6 \\
\pm 1.1\end{array}$ & $\begin{array}{l}13.0 \\
\pm 1.0\end{array}$ & $\begin{array}{l}13.0 \\
\pm 1.0\end{array}$ & $\begin{array}{l}15.6 \\
\pm 0.5\end{array}$ & $\begin{array}{l}15.6 \\
\pm 0.5\end{array}$ & $\begin{array}{l}15.6 \\
\pm 0.5\end{array}$ & $\begin{array}{r}9.3 \\
\pm 1.1\end{array}$ & $\begin{array}{r}9.6 \\
\pm 0.5\end{array}$ & $\begin{array}{r}9.6 \\
\pm 0.5\end{array}$ & $\begin{array}{l}13.6 \\
\pm 0.5\end{array}$ & $\begin{array}{l}18.6 \\
\pm 0.5\end{array}$ & $\begin{array}{l}13.6 \\
\pm 0.5\end{array}$ & $\begin{array}{r}8.0 \\
\pm 1.0\end{array}$ & $\begin{array}{r}8.6 \\
\pm 0.5\end{array}$ & $\begin{array}{r}9.0 \\
\pm 1.0\end{array}$ \\
\hline $\begin{array}{l}\text { X. campestris } \\
\text { PV campestris } \\
(\mathrm{Xcv} 112)\end{array}$ & $\begin{array}{l}15.0 \\
\pm 1.0\end{array}$ & $\begin{array}{l}15.3 \\
\pm 0.5\end{array}$ & $\begin{array}{l}18.0 \\
\pm 1.0\end{array}$ & $\begin{array}{l}13.0 \\
\pm 1.0\end{array}$ & $\begin{array}{l}15.0 \\
\pm 1.0\end{array}$ & $\begin{array}{r}9.6 \\
\pm 0.5\end{array}$ & $\begin{array}{l}17.3 \\
\pm 1.1\end{array}$ & $\begin{array}{l}16.0 \\
\pm 1.0\end{array}$ & $\begin{array}{l}14.6 \\
\pm 1.0\end{array}$ & $\begin{array}{l}13.6 \\
\pm 0.5\end{array}$ & $\begin{array}{l}18.6 \\
\pm 0.5\end{array}$ & $\begin{array}{l}13.6 \\
\pm 0.5\end{array}$ & $\begin{array}{l}16.0 \\
\pm 1.0\end{array}$ & $\begin{array}{l}19.0 \\
\pm 1.0\end{array}$ & $\begin{array}{l}18.6 \\
\pm 1.5\end{array}$ \\
\hline
\end{tabular}

$\mathrm{R}$ - Resistant. No halo formation.

Table 5. Minimum Inhibitory Concentration (MIC) and Minimum Microbicidal Concentration (MMC) results of commercial copaiba oil against clinical interest bacteria

\begin{tabular}{lccccc}
\hline & A1 & A2 & A3 & A4 & A5 \\
\hline E. coli (ATCC 35218) & $\geq 125.0 \mathrm{t}$ & $\geq 125.0 \mathrm{t}$ & $\geq 125.0 \mathrm{t}$ & $\geq 125.0 \mathrm{t}$ & $\geq 250.0 \mathrm{t}$ \\
E. faecalis (ATCC31299) & $\geq 3.90 \mathrm{t}$ & $\geq 31.25 \mathrm{t}$ & $\geq 3.90 \mathrm{t}$ & $\geq 3.90 \mathrm{t}$ & $\geq 250.0 \mathrm{t}$ \\
K. pneumoniae (ATCC700603) & $\geq 250.0 \mathrm{t}$ & $\geq 250.0 \mathrm{t}$ & $\geq 125.0 \mathrm{t}$ & $\geq 125.0 \mathrm{t}$ & $\geq 250.0 \mathrm{t}$ \\
P. aeruginosa (ATCC27853) & $\geq 125.0 \mathrm{t}$ & $\geq 3.90 \mathrm{t}$ & $\geq 3.90 \mathrm{t}$ & $\geq 3.90 \mathrm{t}$ & $\geq 250.0 \mathrm{t}$ \\
S. aureus (ATCC 25921) & $\geq 125.0 \mathrm{t}$ & $\geq 3.90 \mathrm{t}$ & $\geq 3.90 \mathrm{t}$ & $\geq 3.90 \mathrm{t}$ & $\geq 250.0 \mathrm{t}$ \\
S. saprophyticus (ATCC 35552) & $\geq 125.0 \mathrm{t}$ & $\geq 125.0 \mathrm{t}$ & $\geq 250.0 \mathrm{t}$ & $\geq 125.0 \mathrm{t}$ & $\geq 250.0 \mathrm{t}$ \\
\hline
\end{tabular}

$\mathrm{t}$ - bacteriostactic, $\mathrm{f}$ - bactericidal.

commercialized samples which implies adulteration of oil with other oils. Biavatti et al. (2006) discuss that the lack of parameters to characterise and, so far, demand of the quality control certification is an important problem to get registered and to export these products.
The diterpene copalic acid has been considered a marker compound of the Copaifera spp. oils. The kolavenic, kaurenoic and hardwickiic acids are also often described (Veiga et al., 1997; Veiga and Pinto, 2002). Differences in the chromatographic profile of 
Fonseca, R. G., Barros, F. M., Apel, M. A., von Poser, G. L., Andriolli, J. L., Filho, P. C. C., Sousa, D. F., Lobo, I. P., Conceição, A. O. (2015). Physicochemical and antimicrobial properties of copaiba oil: implications on product quality control. Acta Sci. Pol. Technol. Aliment., 14(3), 215-225. DOI: 10.17306/J.AFS.2015.3.23

Table 6. Minimum Inhibitory Concentration (MIC) and Minimum Microbicidal Concentration (MMC) results of commercial copaiba oil against phytopathogens bacteria

\begin{tabular}{lccccc}
\hline & A1 & A2 & A3 & A4 & A5 \\
\hline Acidovorax citrulli (Aac. 1.12) & $\geq 15.62 \mathrm{t}$ & $\geq 125.0 \mathrm{t}$ & $\geq 250.0 \mathrm{f}$ & $\geq 250.0 \mathrm{f}$ & $\geq 250.0 \mathrm{f}$ \\
Pectobacterium caratovorum subsp. caratovorum (Pcc23) & $\geq 125.0 \mathrm{t}$ & $\geq 62.5 \mathrm{t}$ & $\geq 125.0 \mathrm{t}$ & $\geq 125.0 \mathrm{t}$ & $\geq 250.0 \mathrm{f}$ \\
Ralstonia solanacearum (B19) & $\geq 125.0 \mathrm{t}$ & $\geq 3.90 \mathrm{t}$ & $\geq 125.0 \mathrm{t}$ & $\geq 3.90 \mathrm{t}$ & $\geq 250.0 \mathrm{t}$ \\
Ralstonia solanacearum (CGH12) & $\geq 250.0 \mathrm{f}$ & $\geq 15.62 \mathrm{t}$ & $\geq 250.0 \mathrm{f}$ & $\geq 250.0 \mathrm{f}$ & $\geq 250.0 \mathrm{f}$ \\
Xanthomonas campestris PV campestris (Xcc56) & $\geq 125.0 \mathrm{f}$ & $\geq 125.0 \mathrm{f}$ & $\geq 250.0 \mathrm{t}$ & $\geq 125.0 \mathrm{f}$ & $\geq 250.0 \mathrm{t}$ \\
Xanthomonas campestris PV campestris (Xcv112) & $\geq 250.0 \mathrm{f}$ & $\geq 15.62 \mathrm{t}$ & $\geq 125.0 \mathrm{f}$ & $\geq 125.0 \mathrm{f}$ & $\geq 250.0 \mathrm{t}$ \\
\hline
\end{tabular}

$\mathrm{t}$ - bacteriostactic, $\mathrm{f}$ - bactericidal.

Table 7. Volatile content of A1 copaiba oil sample

\begin{tabular}{|c|c|c|c|}
\hline Retention time & Kovats index & Compound & Percentage \\
\hline 24.03 & 1365 & $\alpha$-copaene & 6.32 \\
\hline 24.74 & 1381 & 7-epi-sesquitujene & 3.98 \\
\hline 25.88 & 1406 & $\beta$-caryophyllene & 10.27 \\
\hline 26.70 & 1424 & $Z$ - $\alpha$-bergamotene & 25.65 \\
\hline 27.00 & 1430 & $Z$ - $\beta$-farnesene & 0.71 \\
\hline 27.35 & 1438 & $\alpha$-humulene & 0.69 \\
\hline 27.65 & 1445 & $E$ - $\beta$-farnesene & 2.04 \\
\hline 28.37 & 1461 & $\gamma$-muurolene & 1.59 \\
\hline 28.54 & 1464 & germacrene D & 0.64 \\
\hline 28.73 & 1469 & NI & 2.66 \\
\hline 29.54 & 1486 & $Z$ - $\alpha$-bisabolene & 1.12 \\
\hline 29.83 & 1493 & $\beta$-bisabolene & 29.95 \\
\hline 30.35 & 1505 & $\delta$-amorphene $+\mathrm{NI}$ & 6.76 \\
\hline 31.20 & 1528 & NI & 1.46 \\
\hline 50.14 & 2055 & NI & 0.09 \\
\hline 50.19 & 2056 & NI & 0.09 \\
\hline 53.11 & 2154 & NI & 0.29 \\
\hline 59.37 & 2385 & copalic acid & 0.81 \\
\hline 65.69 & 2626 & NI & 1.21 \\
\hline \multirow[t]{2}{*}{65.76} & 2629 & NI & 0.13 \\
\hline & & & 96.46 \\
\hline
\end{tabular}

$\mathrm{NI}$ - not identified. 
the investigated oils (Fig. 1) suggest adulteration, possibly soybean oil in accordance with literature data (Barbosa et al., 2009; Vasconcelos and Goldinho, 2002; Veiga and Pinto, 2002; Veiga et al., 1997). Thus, the results from physicochemical analysis reinforce the fact that it is imperative to include the analysis of copaiba oil which will ensure the quality of commercialized products. On the other hand, the similarity of sample A1 with in natura copaiba oil was confirmed by GC-MS analysis with $\alpha$-copaene, $\beta$-caryophyllene, $Z$ - $\alpha$-bergamotene, $\alpha$-humulene and $\beta$-bisabolene (Table 5) being among the main sesquiterpenes of copaiba oleoresins (Leandro et al., 2012).

The antimicrobial findings associated to variable quality of samples representing products from Copaifera spp. indicate that public measures of pharmacosurveillance should be the priority of the executive secretariat of the drug market regulation chamber to avoid antimicrobial resistance strain selection as well as to have a suitable product to supply the international market.

\section{REFERENCES}

Adams, R. P. (2009). Identification of essential oil components by gas chromatography/mass spectrometry. Illinois: Allured.

Amaral, A. C. F., Simões, E. V., Ferreira, J. L. P. (2005). Coletânea científica de plantas de uso medicinal. Scientific collection of medicinal plants. Rio de Janeiro, Brasil: FIOCRUZ.

Barbosa, K. S., Yoshida, M., Scudeller, V. V. (2009). Detection of adulterated copaiba (Copaifera multijuga Hayne) oil-resins by refractive index and thin layer chromatography. Rev. Brasil. Farmac., 19, 1A, 57-60.

Barbosa, P. C. S., Medeiros, R. S., Sampaio, P. T. B., Vieira, G., Wiedemann, L. S. M., V.-J., Valdir, F. (2012). Influence of abiotic factors on the chemical composition of copaiba oil (Copaifera multijuga Hayne): soil composition, seasonality and diameter at breast height. J. Braz. Chem. Soc., 23, 1823-1833.

Biavatti, M. W., Dossin, D., Deschamps, F. C., Lima, M. P. (2006). Análise de óleos-resinas de copaíba: contribuição para o seu controle de qualidade [Copaiba oil-resin analysis: contribution to quality control]. Rev. Bras. Farmac., 16, 2, 230-235.

Correia, A. F., Segovia, J. F. O., Gonçalves, M. C. A., de Oliveira, V. L., Silveira, D., Carvalho, J. C. T., Kanzaki,
L. I. B. (2008). Amazonian plant crude extract screening for activity against multidrug-resistant bacteria. Eur. Rev. Med. Pharm. Sci., 12, 369-380.

Deus, R. J. A. D., Alves, C. N., Arruda, M. S. P. (2011). Evaluation of the antifungal effect of oleoresin and essential oil of copaiba (Copaifera multijuga Hayne). Braz. J. Med. Plants, 13, 1-7.

Estevão, L. R. M., Medeiros, J. P., Scognamillo-Szabó, M. V. R., Baratella-Evêncio, L., Guimarães, E. C., Câmara, C. A. G., Evêncio-Neto, J. (2009). Neoangiogenesis of skin flaps in rats treated with copaiba oil. Pesquisa Agropec. Brasil., 44, 406-412.

Leandro, L. M., Vargas, de F. S., Barbosa, P. C. S., Neves, J. K. O., da Silva, J. A., da Veiga-Junior, V. F. (2012). Chemistry and biological activities of terpenoids from copaiba (Copaifera spp.) oleoresins. Molecules, 17, 3866-3889.

Marchesini, A. M., Prado, G. G., Messiano, G. B., Machado, M. B., Lopes, L. M. X. (2009). Chemical constituents of Aristolochia giberti. J. Brazil. Chem. Soc., 20, 1598-1608.

Mendonça, D. E., Onofre, S. B. (2009). Antimicrobial activity of the oil-resin produced by copaiba Copaifera multijuga Hayne (Leguminosae). Braz. J. Pharmac., 19, 2B, 577-581.

Messiano, G. B., Vieira, L., Machado, M. B., Lopes, L. M. X., de Bortoli, S. A., Zukerman-Schpector, J. (2008). Evaluation of insecticidal activity of diterpenes and lignans from Aristolochia malmeana against Anticarsia gemmatalis. J. Agric. Food Chem., 56, 2655-2659.

Oliveira, E. C. P., Lameira, O. A., Zoghbi, M. G. B. (2006). Identification of the time for collecting the oil-resin of copaiba (Copaifera spp.) in the city of Moju, Pará state, Brazil. Braz. J. Med. Plants, 8, 14-23.

Pedroso, R. S., Menezes, R. P., Ferreira, J. C., Penatti, M. P. A., Sá, W. M., Malvino, L. D. S., Candido, R. C., Moreira, T. A. (2014). Antifungal susceptibility of isolates of Candida spp. by disk diffusion and broth microdilution. Biosci. J., 30, 1, 304-311.

Pieri, F. A., Mussi, M. C., Moreira, M. A. S. (2009). Copaiba oil (Copaifera sp.): history, extraction, industrial applications and medicinal properties. Braz. J. Med. Plants, 11, 465-472.

Pieri, F. A., Mussi, M. C. M., Fiorini, J. E., Moreira, A. S., Schneedorf, J. M. (2012). Bacteriostatic effect of copaiba oil (Copaifera officinalis) against Streptococcus mutans. Brazil. Dent. J., 23, 36-38.

Santos, A. O., Ueda-Nakamura, T., Dias Filho, B. P., Veiga Jr., V. F., Pinto, A. C., Nakamura, C. V. (2008). Antimicrobial activity of Brazilian copaiba oils obtained from 
different species of the Copaifera genus. Memór. Inst. Oswaldo Cruz, 103, 277-281.

Sartoratto, A., Machado, A. L. M., Delarmelina, C., Figueira, G. M., Duarte, M. C. T., Rehder, V. L. G. (2004). Composition and antimicrobial activity of essential oils from aromatic plants used in Brasil. Brazil. J. Microbiol., 35, 275-280.

Souza, A. B., Moreira, M. R., Borges, C. H. G., Simão, M. R., Bastos, J. K., de Sousa, J. P. B., ..., Veneziani, R. C. S. (2013). Development and validation of a rapid RPHPLC method for analysis of (-)-copalic acid in copaiba oleoresin. Biomed. Chromat., 27, 280-283.

Tempone, A. G. Sartorelli, P., Teixeira, D., Prado, F. O., Calixto, I. A., Lorenzi, H., Melhem, M. S. C. (2008). Brazilian flora extracts as source of novel antileishmanial and antifungal compounds. Memór. Inst. Oswaldo Cruz, 103, 443-449.

Vasconcelos, A. F. F., Godinho, O. E. S. (2002). Use of classical analytical methods to investigate the authenticity of copaiba oil. Quim. Nova, 25, 6/B, 1057-1060.

Veiga Jr., V. F., Patitucci, M. L., Pinto, A. C. (1997). Authenticity control of commercial copaiba oils by high resolution gas chromatography. Quimica Nova, 20, 612-615.

Veiga Jr., V. F., Pinto, A. C. (2002). The Copaifera L. genus. Quim. Nova, 25, 273-286.

Vieira, R. C., Bombardiere, E., Oliveira, J. J., Lino, J. R. R. S., Brito, L. A. B., Junqueira-Kipnis, A. P. (2008). Influence of Copaifera langsdorffii oil on the repair of a surgical wound in the presence of foreign body. Brazil. J. Vet. Res., 28, 358-366.

Accepted for print - Zaakceptowano do druku: 10.06.2015

Received - Przyjęto: 22.04.2015

For citation - Do cytowania

Fonseca, R. G., Barros, F. M., Apel, M. A., von Poser, G. L., Andriolli, J. L., Filho, P. C. C., Sousa, D. F., Lobo, I. P., Conceição, A. O. (2015). Physicochemical and antimicrobial properties of copaiba oil: implications on product quality control. Acta Sci. Pol. Technol. Aliment., 14(3), 215-225. DOI: 10.17306/J.AFS.2015.3.23 